\title{
Reversal of Sensorimotor Gating Deficits in Brattleboro Rats by Acute Administration of Clozapine and a Neurotensin Agonist, but not Haloperidol: a Potential Predictive Model for Novel Antipsychotic Effects
}

\author{
David Feifel*,', Gilia Melendez' and Paul D Shilling' \\ 'Department of Psychiatry, University of California, San Diego, San Diego, CA, USA
}

\begin{abstract}
Prepulse inhibition (PPI) of acoustic startle is decreased in unmedicated schizophrenia patients and similar deficits can be induced in rats through pharmacological, environmental, or neuroanatomical manipulations. Recently, we reported that Brattleboro (BB) rats, a Long Evans (LE) strain with a single gene mutation, have inherent deficits in PPI homologous to those observed in schizophrenia patients. We also reported that PPI deficits in BB rats could be reversed by chronic but not acute administration of $0.5 \mathrm{mg} / \mathrm{kg}$ haloperidol. No other dose or drug was tested in that experiment. In this study, we tested the effects of acute subcutaneous administration of several doses of haloperidol as well as the second-generation antipsychotic, clozapine, and the putative novel antipsychotic, PDI49I63, a neurotensin mimetic that crosses the blood-brain barrier. Consistent with our previous report, BB rats exhibited PPI deficits compared to LE rats and none of the doses of haloperidol produced a significant effect on this PPI deficit. In contrast, 10 and $15 \mathrm{mg} / \mathrm{kg}$ of clozapine and all the doses of PDI49I63 tested reversed the PPI deficits in BB rats. In addition, haloperidol, but not clozapine or PDI49|63 produced significant catalepsy in BB rats, supporting the notion that PDI49I63 has a profile consistent with atypical antipsychotics and providing support for the predictive validity of the PPI results. These results further strengthen the notion that the BB rat is a useful predictive model of antipsychotic efficacy and suggest that this model may differentiate between antipsychotics belonging to different therapeutic categories, for example, first- and second-generation agents.
\end{abstract}

Neuropsychopharmacology (2004) 29, 73 I-738, advance online publication, 4 February 2004; doi: I 0.1038/sj.npp. I 300378

Keywords: antipsychotics; Brattleboro rats; prepulse inhibition; clozapine; neurotensin; schizophrenia

\section{INTRODUCTION}

Prepulse inhibition (PPI) of the acoustic startle reflex is the reduction in the startle response when the startle-eliciting stimulus is immediately preceded by a weak stimulus (Swerdlow and Geyer, 1998). PPI, an operational measure of sensorimotor gating, is deficient in schizophrenia patients, a phenomenon thought to reflect a disruption in corticostriatal-pallidal-pontine circuits involved in preconscious processing of environmental stimuli (Geyer et al, 2001). PPI deficits analogous to those seen in schizophrenia (Braff and Geyer, 1990) can be induced in rats by administering psychomimetic drugs of several different pharmacological

\footnotetext{
*Correspondence: Dr D Feifel, Department of Psychiatry, University of California, San Diego, 200 West Arbor Drive, San Diego, CA $92103-$ 8218, USA, Tel: + I 619543 2485; Fax: + I 6195433738 ,

E-mail: dfeifel@ucsd.edu

Received 08 July 2003; revised 30 October 2003; accepted 04 December 2003

Online publication: 16 December 2003 at http://www.acnp.org/ citations/Npp | 2 16030330 I/default.pdf
}

families including dopamine agonists such as amphetamine and apomorphine (Mansbach et al, 1988), serotonin agonists such as DOI (Sipes and Geyer, 1994), and noncompetitive NMDA antagonists such as phencyclidine (PCP) and dizocilpine (MK801) (Mansbach and Geyer, 1989). Antipsychotics can reverse this disruption, making PPI the basis of a predictive model for antipsychotic drugs (Geyer et al, 2001). PPI deficits produced by dopamine agonists can be reversed by first-generation or 'typical' as well as second-generation or 'atypical' antipsychotics; therefore, this paradigm is not useful in distinguishing between these two categories of antipsychotics. In contrast, PPI deficits produced by serotonin agonists and NMDA antagonists tend to be preferentially reversed by atypical antipsychotics (Geyer et al, 2001). Therefore, the reversal of serotonin agonist and NMDA antagonist-induced PPI disruption may be predictive of atypical antipsychotic drug properties.

Paradigms requiring drugs to induce PPI deficits have certain inherent limitations as models of sensorimotor gating deficits in humans and predictive models of 
antipsychotic potential. Therefore, there is significant interest in development of nonpharmacological paradigms of PPI deficits. Two of the most studied examples of nonpharmacological paradigms of PPI deficits are social isolation-rearing (Geyer et al, 1993) and neonatal hippocampal lesions (Lipska et al, 1995). PPI deficits produced by social isolation rearing are reversed by both typical and atypical antipsychotics (Cilia et al, 2001; Le Pen and Moreau, 2002). Preliminary findings suggest that PPI deficits produced by neonatal hippocampal lesions may be reversed by atypical but not typical antipsychotics (Le Pen and Moreau, 2002).

Brattleboro (BB) rats are Long Evans (LE) rats with a single base pair mutation that results in the inability to properly synthesize the neurotransmitter and neurohormone vasopressin. $\mathrm{BB}$ rats have many behavioral and cognitive abnormalities, including deficits in memory (Laycock et al, 1983), emotion (Williams et al, 1985), social recognition (Engelmann and Landgraf, 1994), motivation, and attention (Williams et al, 1983). They have abnormalities in brain systems including dopamine and serotonin, neurotransmitters implicated in schizophrenia (Feenstra et al, 1990). BB rats also have deficits in PPI compared with their wild-type, LE counterparts. We previously showed that acute treatment with a single dose $(0.5 \mathrm{mg} / \mathrm{kg})$ of haloperidol did not reverse the PPI deficits in BB rats. In contrast, chronic treatment with that dose of haloperidol reverses the deficits, suggesting that the $\mathrm{BB}$ rat may be a very useful genetic model of sensorimotor gating deficits associated with schizophrenia that models the therapeutic time course of antipsychotic drugs (Feifel and Priebe, 2001). Since only one dose of haloperidol was used in that study, it is possible that acute administration of higher or lower doses of haloperidol may reverse the PPI deficits observed in BB rats. Furthermore, it is not known whether haloperidol's inability to reverse PPI deficits in BB rats after acute administration extends to other established or putative antipsychotic drugs. Therefore, we performed dose response experiments to determine if haloperidol, and the atypical antipsychotic, clozapine and the putative antipsychotic, PD149163, a neurotensin mimetic that has been shown in previous studies to display antipsychotic-like properties (Feifel et al, 1999), would attenuate BB PPI deficits. Furthermore, we tested the catalepsy effects of all three drugs in order to determine whether PD149163 resembles clozapine or haloperidol in this respect, and thus to facilitate interpretation of the predictive validity of the PPI results.

\section{METHODS}

All experimental procedures were conducted in accordance with the University of California, San Diego guidelines for animal care and experimentation. In total, 132 male BB rats and $144 \mathrm{LE}$ rats (170-350 g at testing, Harlan Laboratories, San Diego) were housed in groups of two or three in clear plastic chambers in a climate controlled room under a 12/ $12 \mathrm{~h}$ light/dark schedule (lights on/off - 0700/1900). They were allowed free access to food and water for the extent of the study. Behavioral testing was performed 7 days after arrival, during the light phase of the rats' circadian illumination schedule as startle magnitude, PPI, and drug effects on PPI are stable across the circadian cycle (Weiss et al, 1999). The rats were tested in startle chambers to characterize their baseline PPI and startle. Animals were assigned, based on their baseline PPI, to one of four groups matched so as to achieve comparable average PPI across groups. Drug treatment began 3 days after baseline testing.

In one experiment $50 \mathrm{LE}$ rats and $50 \mathrm{BB}$ rats were administered subcutaneous (s.c.) injections of either 0 (vehicle), $0.1,0.5$, or $1 \mathrm{mg} / \mathrm{kg}$ of haloperidol (UCSD Medical Center, San Diego, CA). In another experiment $51 \mathrm{LE}$ and 49 $\mathrm{BB}$ rats were administered s.c. injection of either 0 (vehicle), 5,10 , or $15 \mathrm{mg} / \mathrm{kg}$ clozapine (Sigma Chemicals, St Louis, $\mathrm{MO})$. In a third study, $43 \mathrm{LE}$ rats and $33 \mathrm{BB}$ rats were administered s.c. injection of either 0 (vehicle), $0.5,1$, or $2 \mathrm{mg} / \mathrm{kg}$ PD149163 (LYS(CH2NH)LYS-PRO-TRP-tLE-LEU$\mathrm{OEt}$ ) (SRI International, NIMH chemical synthesis program). Doses were selected based on their demonstrated ability to reverse PPI deficits in other reports (Feifel et al, 1999; Geyer et al, 2001). Vehicle for haloperidol was distilled water and the volume injected was $1 \mathrm{ml} / \mathrm{kg}$. Vehicle for clozapine and PD149163 was $0.1 \mathrm{~N} \mathrm{HCl}$ and half volume $0.9 \%$ saline brought to $\mathrm{pH} 5-6$ with a few drops of $1 \mathrm{~N}$ $\mathrm{NaOH}$. The volume injected for clozapine was $1.5 \mathrm{ml} / \mathrm{kg}$ and for PD149163 was $1 \mathrm{ml} / \mathrm{kg}$. Each treatment group had a minimum of eight rats.

Animals were tested in startle chambers (San Diego Instruments, San Diego, CA) 20 min after drug administration. Once placed in startle chambers, each rat had a 5-min acclimation period. A $65-\mathrm{dB}$ background noise was continuously present throughout the session. The acclimation was followed by a $15 \mathrm{~min}$ PPI test session during which rats were presented with $40 \mathrm{~ms} 120 \mathrm{~dB}$ startle pulses without a prepulse, or pulses preceded $100 \mathrm{~ms}$ by a prepulse of either 4,8 , or $12 \mathrm{~dB}$ above background. These four types of active stimuli were presented in addition to a neutral (no sound) stimuli condition in pseudorandom order with an average of $15 \mathrm{~s}$ between stimuli types.

A startle response was recorded for all stimuli presentations. PPI for each animal was calculated as a percentage of the pulse-alone startle magnitude using the following formula: (1-(startle magnitude after prepulse-pulse pair/ startle magnitude after pulse only) $\times 100$ ). Exploratory analysis of the data was conducted and indicated that PPI deficits in $\mathrm{BB}$ rats were consistently more robust in the first half of the startle sessions. Therefore, PPI data from this first block of stimuli were subjected to the further statistical analysis. To compare treatments groups, PPI data was subjected to a three-way ANOVA in which prepulse intensity was a within-subject factor and strain and treatment (ie drug dose) were between-subject factors. As expected, percent PPI was inversely related to prepulse intensity in all three experiments (main effect of prepulse intensity) and this is a well-established relationship. However, there was no significant two- or three- way interaction of prepulse intensity with any of the drugs tested. Therefore, this term was dropped from the model and the analysis reported is based upon a reduced model examining averaged PPI from all prepulse intensities. Pairwise comparisons were made using a Dunnett's one-tailed test to test the following specific hypotheses: 
1. PPI in untreated BB rats is significantly lower than in untreated LE rats.

2. Treatment with the test drugs facilitates PPI in BB rats but not in LE rats.

3. Treatment of $\mathrm{BB}$ rats with the test drugs restores their PPI to levels of control LE rats.

Data of the acoustic startle response (ASR) to the startle stimuli not preceded by any prepulse were subjected to analysis using a similar two factor ANOVA.

In a separate study to compare the catalepsy effects of each drug, 48 drug-naïve $\mathrm{BB}$ rats were given one of the following SC treatments: saline, $1 \mathrm{mg} / \mathrm{kg}$ haloperidol, $10 \mathrm{mg} /$ $\mathrm{kg}$ clozapine, $15 \mathrm{mg} / \mathrm{kg}$ clozapine, $1 \mathrm{mg} / \mathrm{kg}$ PD149163 or $2 \mathrm{mg} / \mathrm{kg}$ PD149163 ( $n=8$ for all groups). Doses selected were those that produced the greatest effect in the PPI studies. At 30 minutes after s.c. injections, animals were tested using a method described by several other authors (Stanley and Glick, 1976; Costall et al, 1978; Wadenberg, 1996). This method involved placing the forepaws of each rat in an extended position over a pencil that was suspended horizontally $9 \mathrm{~cm}$ above the lab bench. The time spent in this position before the animal moved or corrected itself was considered a measure of catalepsy.

\section{RESULTS}

\section{Haloperidol PPI Experiment}

Figure 1 (top) illustrates PPI results in the haloperidol experiment. There was a main effect of strain $(\mathrm{F}(1,92)=23.3, P<0.001)$ with $\mathrm{LE}$ rats exhibiting higher PPI than BB rats. There was no significant main effect of haloperidol nor was there a significant haloperidol $\times$ strain interaction. PPI exhibited by vehicle-treated LE rats was significantly higher $(P<0.05)$ than $\mathrm{BB}$ rats for all doses of haloperidol. None of the doses of haloperidol significantly increased PPI in either LE or BB rats.

Figure 1 (bottom) illustrates the ASR results in the haloperidol experiment. Analysis of the ASR data indicates that $\mathrm{BB}$ rats had significantly higher ASR $(\mathrm{F}(1,92)=31.6$, $P<0.001$ ), and that haloperidol significantly decreased the ASR in both strains of rats $(\mathrm{F}(3,92)=13.6, P<0.001)$. There was no significant strain $\times$ haloperidol interaction.

\section{Clozapine PPI Experiment}

Figure 2 (top) illustrates the PPI results in the clozapine experiment. There was a significant main effect of strain $(\mathrm{F}(1,92)=14.7, P<0.001)$ and a strain $\times$ clozapine interaction $(\mathrm{F}(3,92)=3.4, P<0.05)$, but not a main clozapine effect. LE rats treated with vehicle exhibited PPI that was significantly higher than $\mathrm{BB}$ rats treated with vehicle $(P<0.01)$, but not significantly different than $\mathrm{BB}$ rats treated with any of the doses of clozapine tested. Furthermore, PPI in BB rats treated with vehicle was significantly lower than PPI in BB rats treated with $10 \mathrm{mg} / \mathrm{kg}$ $(P<0.01)$ and $15 \mathrm{mg} / \mathrm{kg}$ dose $(P<0.05)$ of clozapine. In contrast, no dose of clozapine increased PPI in LE rats.

Figure 2 (bottom) illustrates the ASR results in the clozapine experiment. Analysis of the ASR data indicates that there was no significant difference in startle magnitude
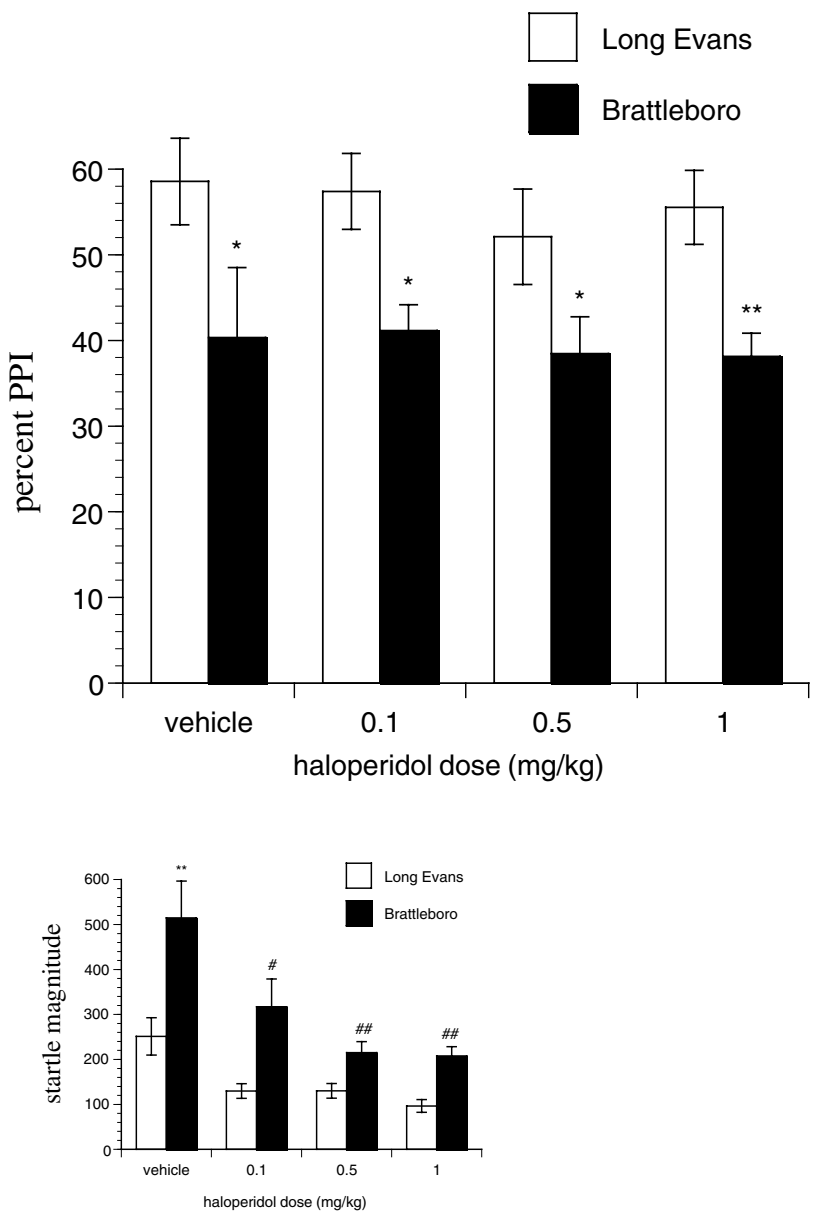

Figure I PPI (top) and ASR (bottom) \pm SEM in rats treated with haloperidol. Significantly different from vehicle-treated LE rats represented by $*(P<0.05)$ and $* *(P<0.01)$. Significantly different from vehicle-treated $\mathrm{BB}$ rats represented by \#\# $(P<0.0 \mathrm{I})$.

between BB and LE rats. Clozapine appeared to reduce ASR in both rat strains in a dose-dependent fashion, but this effect was slightly above the statistical cutoff for significance $(P=0.051)$. There was no significant clozapine $\times$ strain interaction effect.

\section{PD149163 PPI Experiment}

Figure 3 (top) illustrates the PPI results in the PD149163 experiment. There was a significant effect of strain $((\mathrm{F}(1,68)=6.28, \quad P<0.05), \quad$ a significant main effect of PD149163 $(\mathrm{F}(3,68)=4.75, \quad P<0.01)$, and a significant strain $\times$ PD149163 interaction $(F(3,68)=4.221, P<0.01)$. PPI in vehicle-treated LE rats was significantly higher than in vehicle-treated $\mathrm{BB}$ rats $(P<0.01)$, but not significantly different from $\mathrm{BB}$ rats treated with any of the doses of PD149163. BB rats treated with 1 and $3 \mathrm{mg} / \mathrm{kg}$ PD149163 had significantly higher PPI $(P<0.01$ and $P<0.05$, respectively) than PPI exhibited by vehicle-treated BB rats. In contrast, no dose of PD149163 had a significant effect on PPI in LE rats, although there appeared to be a tendency for PD149163 to dose-dependently increase PPI in LE rats.

Figure 3 (bottom) illustrates the ASR data for the PD149163 experiment. There was not a significant differ- 

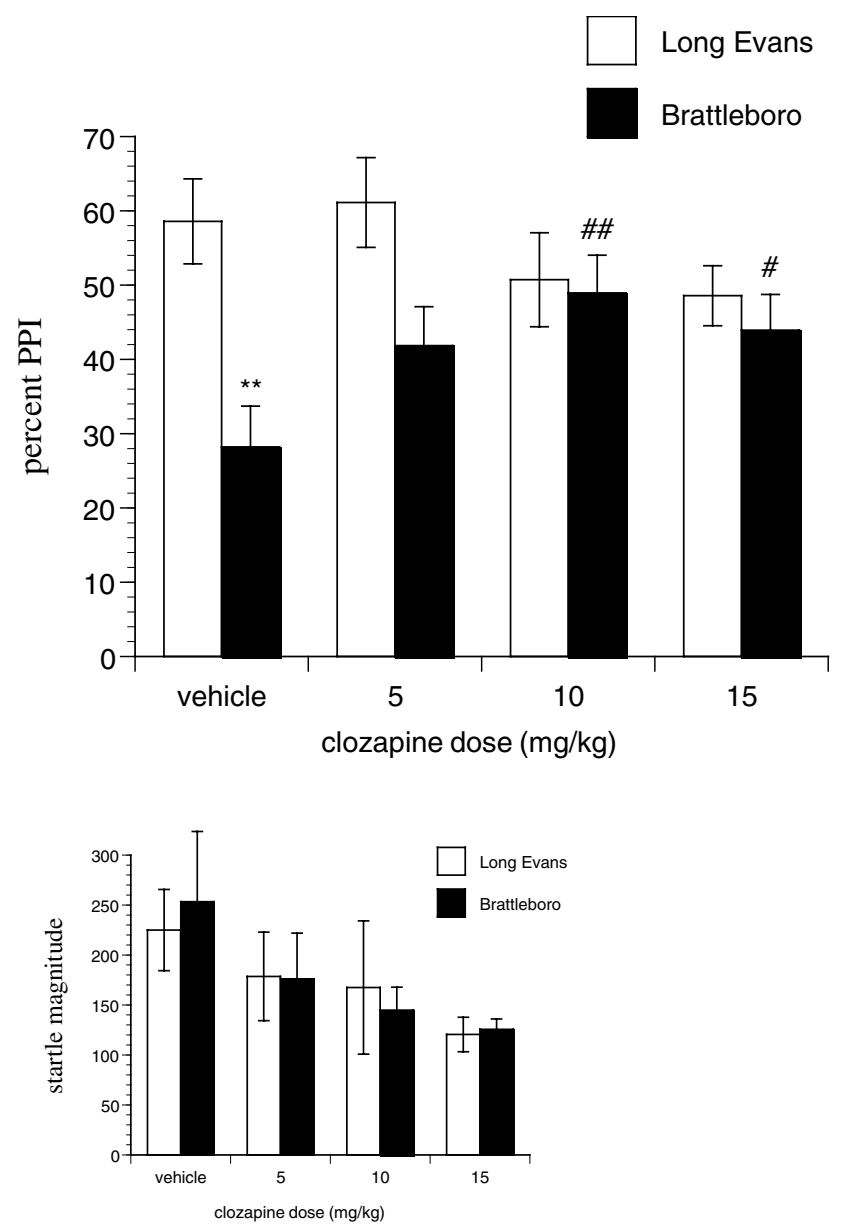

Figure $2 \mathrm{PPI}$ (top) and ASR (bottom) \pm SEM in rats treated with clozapine. Significantly different from vehicle-treated LE rats represented by $* *(P<0.0 \mathrm{I})$. Significantly different from vehicle-treated $\mathrm{BB}$ rats represented by $\#(P<0.05)$.

ence in the ASR between BB and LE rats, but PD149163 significantly decreased the ASR in both strains of rat as evidenced by a main effect of PD149163 $(\mathrm{F}(3,68)=5.3$, $P<0.01)$. There was no significant strain $\times$ PD149163 interaction effect. Each dose of PD149163 significantly reduced $(P<0.05)$ the ASR in BB rats compared to vehicle, whereas the highest dose $(3 \mathrm{mg} / \mathrm{kg})$ reduced the ASR in LE rats compared to vehicle.

\section{Catalepsy Experiment}

Figure 4 illustrates the catalepsy findings. There was a main effect of drug treatment $(\mathrm{F}(5,42)=13.6, P<0.001)$. Post hoc comparisons indicated that haloperidol $(P<0.001)$ but no dose of clozapine or PD149163 significantly increased the time rats remained on the bar.

\section{DISCUSSION}

Consistent with our previous report (Feifel and Priebe, 2001), untreated (vehicle) BB rats exhibited reduced PPI compared to untreated LE rats in each of the three experiments. In our previous study, we reported higher
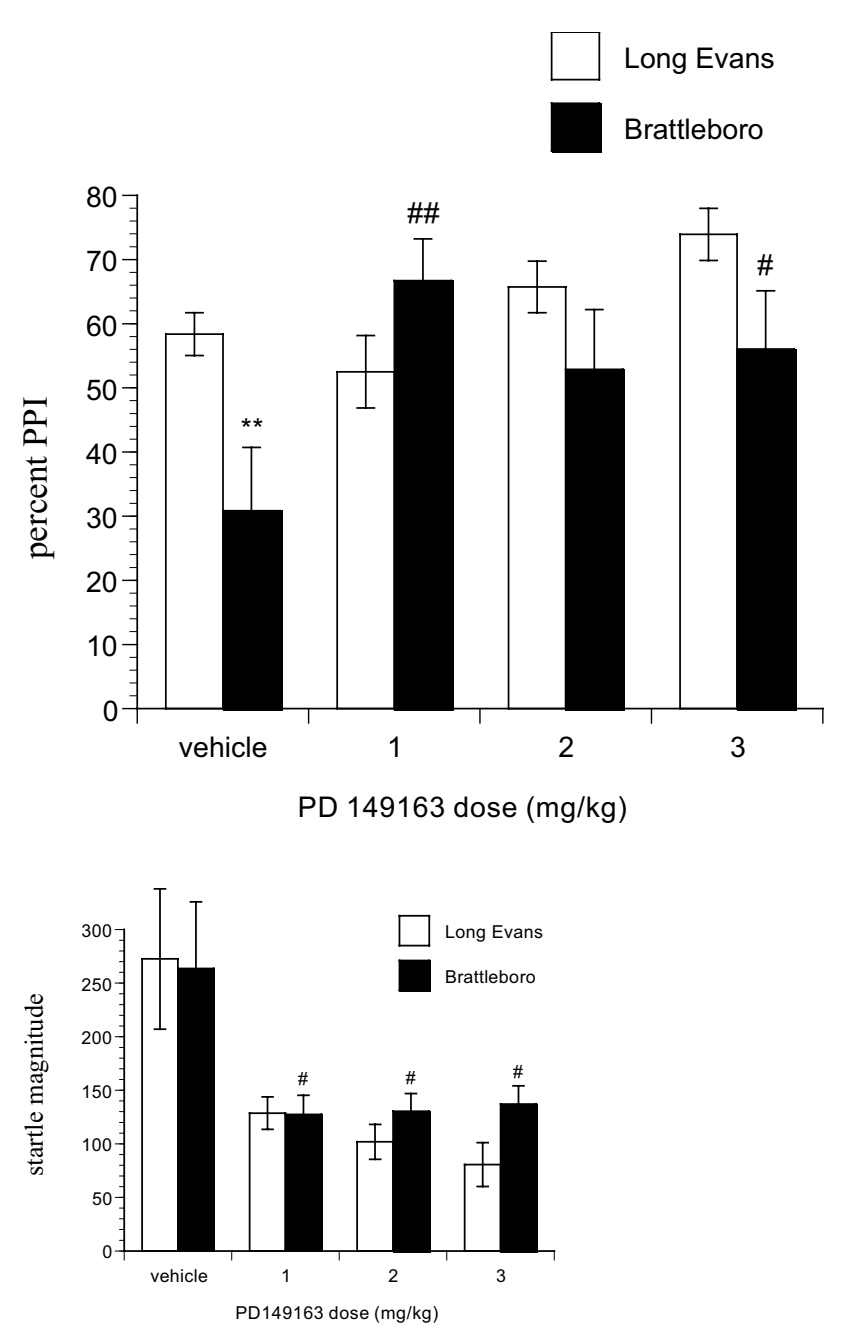

Figure $3 \mathrm{PPI}$ (top) and ASR (bottom) \pm SEM in rats treated with PDI49|69. Significantly different from vehicle-treated LE rats represented by $* *(P<0.01)$. Significantly different from vehicle-treated $B B$ rats represented by $\#(P<0.05)$ and \#\# $(P<0.0$ I $)$.

ASR in BB rats and this effect was also reported by another group (Warren and Gash, 1983). The current data are only partially consistent with those reports since we observed a significantly higher startle response in $\mathrm{BB}$ rats in the haloperidol experiment but not in the clozapine or PD149163 experiment. The reason for this variability in the ASR strain differences is not clear; however, it indicates that there is a disassociation between the ASR strain differences and the PPI strain differences, an observation we also made in our original report. This is also supported by the fact that all three drugs tended to decrease ASR in LE and $\mathrm{BB}$ rats with efficacy that did not correspond to their PPI effects.

Acute administration of haloperidol did not affect PPI in either LE or BB rats, which is also consistent with our previous report. Control (vehicle-treated) rats in the haloperidol group exhibited PPI that was higher (approximately $40 \%$ ) than the control groups in the clozapine and PD149163 experiments (approximately 30\%). However, it is unlikely that this difference contributed to the negative drug effects in the haloperidol experiment since an analysis of 


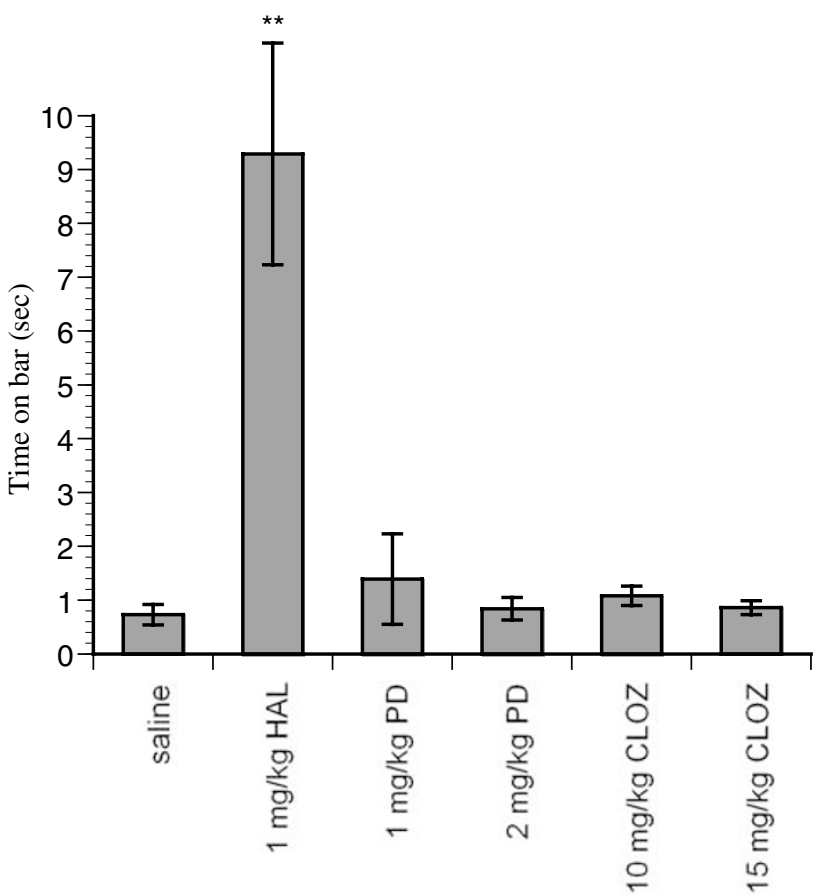

Figure 4 Catalepsy scored as the time paws of rats remained on the bar in seconds $( \pm$ SEM). Significantly different from saline group represented by $* *(P<0.0 \mathrm{I})$.

PPI produced by the weakest prepulse tested $(4 \mathrm{~dB})$, which produced a mean PPI of $26 \%$ in the control group, was not increased by any of the haloperidol doses (PPI of 25\%, 28\%, and $28 \%$ for low, mid and high dose haloperidol, respectively).

In our earlier study, we tested only a single dose of haloperidol $(0.5 \mathrm{mg} / \mathrm{kg})$ and therefore we could not rule out the possibility that higher or lower doses would be more effective. In this experiment, higher and lower doses in addition to the $0.5 \mathrm{mg} / \mathrm{kg}$ dose were tested. Since none of the doses of haloperidol had a significant effect, we can reasonably conclude that the PPI deficit in $\mathrm{BB}$ rats is not affected by acute administration of haloperidol. However, it is known from our previous study that chronic haloperidol reverses PPI deficits in BB rats (Feifel and Priebe, 2001). The lack of an effect after acute administration of any dose of haloperidol in this study confirms that differences in efficacy between chronic and acute haloperidol treatment are due to the temporal aspects of the two treatments, rather than merely choice of dose.

Haloperidol is a prototype of the first generation, or 'typical' family of antipsychotic drugs. These antipsychotics produce their therapeutic effects via a single pharmacological mechanism, antagonism of D2 receptors (Feldman and Quenzer, 1997). Haloperidol's lack of efficacy also indicates that excessive dopamine transmission is not likely to fully account for the PPI deficits seen in BB rats. Typical antipsychotics have notable limitations with respect to clinical efficacy. For example, a significant proportion of schizophrenia patients fail to respond adequately to haloperidol and other antipsychotics from the typical family (Stern et al, 1994). Furthermore, experience with haloperidol and other typical antipsychotics indicates that they have good efficacy against 'positive' symptoms of schizo- phrenia (ie hallucinations, delusions), but they are less efficacious against 'negative' symptoms (ie paucity of thought, decreased emotional expression, decreased volition behavior) or the cognitive deficits known to be associated with the disease (Meltzer, 2002). More recently, a second generation of antipsychotics has been developed. These 'atypical' antipsychotics, of which clozapine is the prototype, appears to be more efficacious, particularly in ameliorating negative symptoms and cognitive deficits associated with schizophrenia (Kinon and Lieberman, 1996). Preclinical paradigms that can differentiate atypical from typical clinical profiles are needed since advances in the therapeutic field make it no longer desirable to develop compounds with first generation clinical profiles. Since it is established that atypical antipsychotics can produce desirable clinical effects not associated with typical antipsychotics, it is reasonable to assume that there exist preclinical paradigms that model this atypical antipsychotic clinical advantage. Thus, whereas sensitivity to haloperidol was once considered the 'litmus test' for validating preclinical models of antipsychotic potential, it is now desirable to develop preclinical models that are preferentially sensitive to atypical antipsychotics over typical antipsychotics such as haloperidol. Indeed, several preclinical paradigms have been proposed to be useful for identifying putative antipsychotics of atypical category. Typically in these paradigms, second-generation antipsychotics have a spectrum of effects that is distinct or broader than typical antipsychotics. Examples of these effects include antagonism of PPI disruption produced by NMDA antagonists (Geyer et al, 2001) and induction of a distinct regional pattern of immediate early gene expression (Deutch and Duman, 1996). In this respect, it is significant that clozapine was able to reverse PPI deficits in BB rats. This reversal was dose dependent with the 10 and $15 \mathrm{mg} / \mathrm{kg}$ dose exhibiting the greatest efficacy. It is not likely that clozapine's ability to acutely reverse PPI deficits in BB rats is due to a nonspecific pharmacological effect not associated with its therapeutic mechanism, sedation for example. Whereas nonspecific effects such as sedation typically reduce normal behavior, for example, locomotor activity, and can thus appear similar to the specific pharmacological effects of antipsychotics, restoration of deficient process, particularly an information processing deficit such as PPI, is unlikely to be produced by a nonspecific effect. Consistent with this notion, Depoortere et al (1997) concluded that clozapine's enhancement of PPI was not likely due to its sedating properties since sedating psychotropic drugs that do not have antipsychotic properties, for example, diazepam, decrease rather than facilitate PPI (Depoortere et al, 1997).

The fact that clozapine but not haloperidol reversed PPI deficits in BB rats suggests that acute reversal of PPI deficits in $\mathrm{BB}$ rats may also have utility as a predictive model for atypical antipsychotic-like properties among putative antipsychotics. While there is some debate as to what pharmacological properties underlie the clinical advantages associated with atypical antipsychotics, there is general agreement that combination of antagonism at both the 5HT2A and D2 receptors is vital (Meltzer, 2002). The pattern of PPI effects observed with haloperidol and clozapine in this and other studies may be understood if inhibition of dopamine-2 transmission is sufficient to 
reverse $\mathrm{BB}$ deficits chronically, and that other pharmacological mechanisms, for example, 5HT2 antagonism, potentiate the D2 inhibition to produce a stronger, and thus more rapid reversal of PPI deficits. In this way, inhibition of D2 transmission may be necessary and sufficient for chronic reversal of PPI deficits in BB rats and necessary but not sufficient for acute reversal.

However, other explanations are possible. For example, in addition to sharing clinical properties with all other atypical antipsychotics, clozapine is also considered by many clinicians and investigators to be singular among antipsychotics in regards to efficacy (Taylor and DuncanMcConnell, 2000; Chakos et al, 2001; Conley and Kelly, 2001). This is particularly evident in the high success rates with clozapine among treatment-resistant patients with schizophrenia (Kane et al, 1988; Chakos et al, 2001; Kane et al, 2001). Thus, rather than due to mechanisms shared by all atypical antipsychotics (eg D2 and 5HT2 antagonism), clozapine's ability to abolish PPI deficits in BB rats may be due to a putative mechanism which distinguishes clozapine from all other currently available antipsychotics, and which is responsible for its unique efficacy profile. If this is the case, acute reversal of PPI deficits in BB rats may be a model for drugs with novel properties that, like clozapine, are associated with a superior level of efficacy to current typical and atypical antipsychotics. Studies with other typical and atypical antipsychotic drugs will be important in order to determine whether PPI deficits in the $\mathrm{BB}$ rats is a predictive model of the clinical effects associated with atypical antipsychotics or of novel drugs useful in treatment refractory schizophrenia.

PD149163 appeared to produce the most robust effects on PPI reversing PPI strain differences at all doses tested even though it appeared to produce a nonsignificant tendency to increase PPI in LE rats. PD149163's ability to reverse the PPI deficits in $\mathrm{BB}$ rats is consistent with the notion that the $\mathrm{BB}$ rat is a useful predictive model of antipsychotic efficacy. Significant evidence exists that neurotensin, a neuropeptide, may act as an endogenous antipsychotic and that it may, in fact, mediate some of the clinical effects of antipsychotic drugs (Kinkead and Nemeroff, 2002). There is also a large body of evidence that neurotensin and neurotensin agonists produce antipsychotic-like effects in preclinical studies. This has led many investigators to propose that neurotensin agonists may have clinical potential as antipsychotic drugs (for a review, see Caceda et al, 2003). We have previously shown that administration of neurotensin produces antipsychotic-like effects on PPI (Feifel et al, 1997). The mechanism implicated in the antipsychotic-like effects produced by neurotensin has been inhibition of dopamine transmission in the mesolimbic pathway, although neurotensin does not have significant affinity for dopamine receptors (Adachi et al, 1990; Nouel et al, 1992). The 8-13 amino-acid fragment of neurotensin is the smallest fragment that retains full biological activity of the parent tridecapeptide (Kanba et al, 1988). PD149163 was developed by modifying neurotensin (8-13) to make it more stable to endopeptidase degradation and has been shown to cross the blood-brain barrier after parenteral administration (Wustrow et al, 1995). We have previously shown that PD149163 antagonizes amphetamine-induced disruption of PPI (Feifel et al, 1999). PD149163 also antagonized the PPI disrupting effects of the NMDA antagonist, MK801 (Feifel et al, 1999), and DOI, a 5HT2A agonist (Feifel et al, 2003). These results suggested that PD149163 produces antipsychotic-like preclinical effects by mechanisms other than inhibition of dopamine transmission alone.

In the current study, PD149163 produced a very distinct reversal of PPI deficits in $\mathrm{BB}$ rats. In fact, after treatment with the lowest dose, PPI in BB rats was higher than LE rats, although the difference did not reach statistical significance. The current results also provide further evidence that indirect inhibition of dopamine transmission cannot account, by itself, for the antipsychotic-like effects of PD149163, since PD149163 was effective in reversing BB rat PPI deficits, whereas haloperidol, a potent D2 antagonist, was not. In this study, PD149163's effect on BB PPI was more consistent with clozapine, than with haloperidol. While this finding does not by itself suggest that PD149163 has potential as an antipsychotic, the current finding is consistent with previous evidence suggesting that PD149163 has a preclinical profile consistent with atypical antipsychotic drugs (Feifel et al, 2003). In this respect, PD149163's effects on PPI in BB rats supports the notion that the $\mathrm{BB}$ rat is a predictive model for antipsychotics with atypical or novel mechanisms. The fact that PD149163, like clozapine, did not produce significant catalepsy, whereas haloperidol did, supports the contention that PD149163 has a profile more similar to atypical than typical antipsychotics and strengthens the evidence that acute PPI reversal in BB rats has predictive validity for drugs with atypical antipsychotic effects.

In terms of establishing the validity of the BB model as predictive screen for atypical antipsychotic drugs, these findings should be considered preliminary. Additional studies with other antipsychotics in the BB model will be needed to address the issue of whether PD149163's effects are shared by other atypical antipsychotics and are thus suggestive of 'atypical' clinical features or whether PD149163's effects are shared only by clozapine and are thus suggestive of a uniquely superior efficacy similar to clozapine. It is noteworthy that PD149163 produced the most robust reversal of PPI deficits in BB rats of the three compounds tested, a finding that is auspicious for the therapeutic potential of drugs that target neurotensin receptors.

In summary, the $\mathrm{BB}$ rat offers a model of sensorimotor gating deficits and a predictive model of antipsychotic potential with many novel and useful features. First, this model does not require pharmacological, environmental, or neuroanotomical manipulations to produce PPI deficits. Rather, PPI deficits homologous to those seen in schizophrenia and other neuropsychiatric disorders are exhibited spontaneously and presumably due to the single gene abnormality associated with these rats. As a genetic model of PPI deficits, the BB rat exhibits greater construct validity for the neuropsychiatric disorders associated with PPI deficits compared to models requiring pharmacological, environmental, or neuroanotomical manipulations to produce PPI deficits. This improved construct validity affords the possibility of using the $\mathrm{BB}$ rats to explore the neurobiological and genetic substrates underlying sensorimotor gating abnormalities that may also underlie the neuropsychiatric conditions associated with such deficits. 
As in the context of acute administration, the BB rat model appears to differentiate typical from nontypical antipsychotics and/or drugs with clozapine-like efficacy from drugs with more conventional efficacy, it may also be a useful predictive screen for novel antipsychotic drugs. Haloperidol's effect on BB PPI is greater after chronic administration than after acute administration (Feifel and Priebe, 2001). Therefore, the BB rat model may also be useful for elucidating the mechanisms underlying the therapeutic time course, which is typically associated with antipsychotic treatment in schizophrenia patients. In this regard, it will be important to examine how the effects of chronic administration of clozapine and PD149163 compare to the effect of acute administration of these drugs on BB PPI deficits.

\section{ACKNOWLEDGEMENTS}

DF is supported by a NIMH grant (MH62451). We are grateful for the NIMH Chemical Synthesis Program and SRI international for providing PD149163. We thank Dr. Neal Swerdlow for assistance with the catalepsy paradigm.

\section{REFERENCES}

Adachi DK, Kalivas PW, Schenk JO (1990). Neurotensin binding to dopamine. J Neurochem 54: 1321-1328.

Braff DL, Geyer MA (1990). Sensorimotor gating and schizophrenia. Human and animal model studies. Arch Gen Psychiatry 47: 181-188.

Caceda R, Kinkead B, Nemeroff CB (2003). Do neurotensin receptor agonists represent a novel class of antipsychotic drugs? Semin Clin Neuropsychiatry 8: 94-108.

Chakos M, Lieberman J, Hoffman E, Bradford D, Sheitman B (2001). Effectiveness of second-generation antipsychotics in patients with treatment-resistant schizophrenia: a review and meta-analysis of randomized trials. Am J Psychiatry 158: $518-526$

Cilia J, Reavill C, Hagan JJ, Jones DN (2001). Long-term evaluation of isolation-rearing induced prepulse inhibition deficits in rats. Psychopharmacology (Berl) 156: 327-337.

Conley RR, Kelly DL (2001). Management of treatment resistance in schizophrenia. Biol Psychiatry 50: 898-911.

Costall B, Hui SC, Naylor RJ (1978). Correlation between multitest and single test catalepsy assessment. Neuropharmacology 17: 761-764.

Depoortere R, Perrault G, Sanger DJ (1997). Potentiation of prepulse inhibition of the startle reflex in rats: pharmacological evaluation of the procedure as a model for detecting antipsychotic activity. Psychopharmacology 132: 366-374.

Deutch AY, Duman RS (1996). The effects of antipsychotic drugs on Fos protein expression in the prefrontal cortex: cellular localization and pharmacological characterization. Neuroscience 70: 377-389.

Engelmann M, Landgraf R (1994). Microdialysis administration of vasopressin into the septum improves social recognition in Brattleboro rats. Physiol Behav 55: 145-149.

Feenstra MG, Snijdewint FG, Van Galen H, Boer GJ (1990). Widespread alterations in central noradrenaline, dopamine, and serotonin systems in the Brattleboro rat not related to the local absence of vasopressin. Neurochem Res 15: 283-288.

Feifel D, Melendez G, Shilling PD (2003). A systemically administered neurotensin agonist blocks disruption of prepulse inhibition produced by a serotonin-2A agonist. Neuropsychopharmacology 28: 651-653.
Feifel D, Minor KL, Dulawa S, Swerdlow NR (1997). The effects of intra-accumbens neurotensin on sensorimotor gating. Brain Res 760: $80-84$.

Feifel D, Priebe K (2001). Vasopressin-deficient rats exhibit sensorimotor gating deficits that are reversed by subchronic haloperidol. Biol Psychiatry 50: 425-433.

Feifel D, Reza TL, Wustrow DJ, Davis MD (1999). Novel antipsychotic-like effects on prepulse inhibition of startle produced by a neurotensin agonist. J Pharmacol Exp Therapeut 288: 710-713.

Feldman RS MJ, Quenzer LF (1997). Principles of Neuropsychopharmacology. Sinauer Associates, Inc.: Sunderland, MA.

Geyer MA, Krebs-Thomson K, Braff DL, Swerdlow NR (2001). Pharmacological studies of prepulse inhibition models of sensorimotor gating deficits in schizophrenia: a decade in review. Psychopharmacology (Berl) 156: 117-154.

Geyer MA, Wilkinson LS, Humby T, Robbins TW (1993). Isolation rearing of rats produces a deficit in prepulse inhibition of acoustic startle similar to that in schizophrenia. Biologic Psychiatry 34: 361-372.

Kanba KS, Kanba S, Nelson A, Okazaki H, Richelson E (1988). $\left[{ }^{3} \mathrm{H}\right]$ neuroten $\sin (8-13)$ binds in human brain to the same sites as does $\left[{ }^{3} \mathrm{H}\right]$ neurotensin but with higher affinity. J Neurochem 50: 131-137.

Kane JM, Honigfeld G, Singer J, Meltzer H (1988). Clozapine in treatment-resistant schizophrenics. Psychopharmacol Bull 24: 62-67.

Kane JM, Marder SR, Schooler NR, Wirshing WC, Umbricht D, Baker RW et al (2001). Clozapine and haloperidol in moderately refractory schizophrenia: a 6-month randomized and doubleblind comparison. Arch Gen Psychiatry 58: 965-972.

Kinkead B, Nemeroff CB (2002). Neurotensin: an endogenous antipsychotic? Curr Opin Pharmacol 2: 99-103.

Kinon BJ, Lieberman JA (1996). Mechanisms of action of atypical antipsychotic drugs: a critical analysis. Psychopharmacology (Berl) 124: 2-34.

Laycock JF, Gartside IB, Chapman JT (1983). A comparison of the learning abilities of Brattleboro rats with hereditary diabetes insipidus and Long-Evans rats using positively reinforced operant conditioning. Prog Brain Res 60: 183-187.

Le Pen G, Moreau JL (2002). Disruption of prepulse inhibition of startle reflex in a neurodevelopmental model of schizophrenia: reversal by clozapine, olanzapine and risperidone but not by haloperidol. Neuropsychopharmacology 27: 1-11.

Lipska BK, Swerdlow NR, Geyer MA, Jaskiw GE, Braff DL, Weinberger DR (1995). Neonatal excitotoxic hippocampal damage in rats causes post-pubertal changes in prepulse inhibition of startle and its disruption by apomorphine. Psychopharmacology 122: 35-43.

Mansbach RS, Geyer MA (1989). Effects of phencyclidine and phencyclidine biologs on sensorimotor gating in the rat. Neuropsychopharmacology 2: 299-308.

Mansbach RS, Geyer MA, Braff DL (1988). Dopaminergic stimulation disrupts sensorimotor gating in the rat. Psychopharmacology 94: 507-514.

Meltzer HY (2002). Mechanism of action of atypical antipsychotic drugs. In: Davis KLC, Joseph Coyle D, Nemeroff C (eds). Neuropsychopharmacology: The Fifth Generation of Progress. American College of Neuropsychopharmacology, Lippincot, Philadelphia.

Nouel D, Costentin J, Lugrin D, Kitabgi P, Ple N, Davoust D (1992). Investigations about a direct neurotensin-dopamine interaction by nuclear magnetic resonance study, synaptosomal uptake of dopamine, and binding of neurotensin to its receptors. J Neurochem 59: 1933-1936.

Sipes TA, Geyer MA (1994). Multiple serotonin receptor subtypes modulate prepulse inhibition of the startle response in rats. Neuropharmacology 33: 441-448. 
Stanley ME, Glick SD (1976). Interaction of drug effects with testing procedures in the measurement of catalepsy. Neuropharmacology 15: 393-394.

Stern RG, Kahn RS, Davidson M, Nora RM, Davis KL (1994). Early response to clozapine in schizophrenia. Am J Psychiatry 151: $1817-1818$.

Swerdlow NR, Geyer MA (1998). Using an animal model of deficient sensorimotor gating to study the pathophysiology and new treatments of schizophrenia. Schizophrenia Bulletin 24: 285-301.

Taylor DM, Duncan-McConnell D (2000). Refractory schizophrenia and atypical antipsychotics. J Psychopharmacol 14: 409-418.

Wadenberg ML (1996). Serotonergic mechanisms in neurolepticinduced catalepsy in the rat. Neurosci Biobehav Rev 20: 325-339.
Warren PH, Gash DM (1983). Hyperreflexive behavior in Brattleboro rats. Peptides 4: 421-424.

Weiss IC, Feldon J, Domeney AM (1999). Circadian time does not modify the prepulse inhibition response or its attenuation by apomorphine. Pharmacol, Biochem Behav 64: 501-505.

Williams AR, Carey RJ, Miller M (1983). Behavioral differences between vasopressin-deficient (Brattleboro) and normal LongEvans rats. Peptides 4: 711-716.

Williams AR, Carey RJ, Miller M (1985). Altered emotionality of the vasopressin-deficient Brattleboro rat. Peptides 6(Suppl 1): 69-76.

Wustrow DJ DM, Akunne HC, Corbin AE, Wiley JN, Wise LD, Heffner TG (1995). Reduced amide bond neurotensin 8-13 mimetics with potent in vivo activity. Bioorgan Medicin Chem Lett 5: 997-1002. 